\title{
Are evidence-based guidelines reflected in clinical practice? An analysis of prospectively collected data of the Italian Thyroid Cancer Observatory (ITCO\#1).
}

\author{
Lamartina L, Durante C, Lucisano G, Grani G, Bellantone R, Lombardi CP, Pontecorvi A, \\ Arvat E, Felicetti F, Zatelli MC, Rossi R, Puxeddu E, Morelli S, Torlontano M, Crocetti U, \\ Montesano T, Giubbini R, Orlandi F, Aimaretti G, Monzani F, Attard M, Francese C, \\ Antonelli A, Limone P, Rossetto R, Fugazzola L, Meringolo D, Bruno R, Tumino S, Ceresini G, \\ Centanni M, Monti S, Salvatore D, Spiazzi G, Mian C, Persani L, Barbaro D, Nicolucci A and \\ Filetti S
}

\begin{abstract}
These data were presented at the $39^{\text {th }}$ European Thyroid Association Meeting as oral communication.
\end{abstract}

Livia Lamartina, MD, Department of Internal Medicine and Medical Specialties, University of Rome Sapienza, Rome, Italy. livia.lamartina@uniroma1.it

Cosimo Durante, MD, PhD, Department of Internal Medicine and Medical Specialties, University of Rome Sapienza, Rome, Italy. cosimo.durante@uniroma1.it Giuseppe Lucisano, Center for Outcomes Research and Clinical Epidemiology, Pescara, Italy. lucisano@coresearch.it

Giorgio Grani, MD, Department of Internal Medicine and Medical Specialties, University of Rome Sapienza, Rome, Italy. giorgio.grani@uniroma1.it

Rocco Bellantone, Division of Endocrine Surgery, Fondazione Policlinico Gemelli, Catholic University, Rome, Italy. rocco.bellantone@unicatt.it

Celestino Pio Lombardi, Division of Endocrine Surgery, Fondazione Policlinico Gemelli, Catholic University, Rome, Italy. celestinopio.Lombardi@unicatt.it

Alfredo Pontecorvi, Division of Endocrinology and Metabolic Diseases, Fondazione Policlinico Gemelli, Catholic University, Rome, Italy. alfredo.pontecorvi@unicatt.it Emanuela Arvat, Department of Medical Sciences, University of Turin, Turin, Italy. emanuela.arvat@unito.it

Francesco Felicetti, Department of Medical Sciences, University of Turin, Turin, Italy. franci.feli@libero.it 
Maria Chiara Zatelli, Endocrine Unit, Azienda Ospedaliero Universitaria S. Anna, Ferrara. Ferrara.ztImch@unife.it

Roberta Rossi, Endocrine Unit, Azienda Ospedaliero Universitaria S. Anna, Ferrara. roberta.rossi@unife.it

Efisio Puxeddu, Department of Medicine, University of Perugia, Perugia, Italy. efisio.puxeddu@unipg.it

Silvia Morelli, Department of Medicine, University of Perugia, Perugia, Italy. silviettamorelli@gmail.com

Massimo Torlontano, Department of Medical Science, Ospedale Casa Sollievo della Sofferenza-IRCCS, San Giovanni Rotondo, Italy.m.torlontano@operapadrepio.it Umberto Crocetti, Department of Medical Science, Ospedale Casa Sollievo della Sofferenza-IRCCS, San Giovanni Rotondo, Italy. u.crocetti@operapadrepio.it Teresa Montesano, Department of Nuclear Medicine, University of Rome Sapienza, Rome, Italy. teresa.montesano@uniroma1.it

Raffaele Giubbini, Nuclear Medicine Unit, Spedali Civili Università degli Studi di Brescia, Brescia, Italy.giubbini@med.unibs.it

Fabio Orlandi, Department of Oncology, University of Turin. Division of Endocrinology and Metabolism, Humanitas-Gradenigo Hospital, Turin, Italy. fabio.orlandi@unito.it Gianluca Aimaretti, Endocrinology. Department of Translational Medicine, Università del Piemonte Orientale, Novara, Italy.gianluca.aimaretti@med.uniupo.it Fabio Monzani, Geriatrics Unit, Department of Clinical \& Experimental Medicine, University of Pisa, Pisa, Italy. fabio.monzani@med.unipi.it Marco Attard, Division of Endocrinology, Cervello Hospital, Palermo, Italy. mattard@libero.it

Cecilia Francese, Division of Endocrinology, Salerno, Italy. cecilia.francese@alice.it Alessandro Antonelli, Department of Clinical and Experimental Medicine, University of Pisa, Pisa, Italy. alessandro.antonelli@med.unipi.it

Paolo Limone, Division of Endocrinology, Diabetology and Metabolism, Mauriziano Umberto I Hospital, Turin, Italy. plimone@mauriziano.it 
Ruth Rossetto, Division of Endocrinology, Diabetology and Metabolism, Department of Medical Sciences, Molinette Hospital, A.O.U. Città della Salute e della Scienza di Torino, University of Turin, Turin, Italy. ruth.rossetto@unito.it Laura Fugazzola, Division of Endocrine and Metabolic Diseases, IRCCS Istituto Auxologico Italiano, Milan, and Department of Pathophysiology and Transplantation, Università degli Studi di Milano, Italy. laura.fugazzola@unimi.it

Domenico Meringolo, Unità Operativa Semplice Dipartimentale di Endocrinologia, Ospedale di Bentivoglio, Bologna, Italy. meringolod@libero.it

Rocco Bruno, Unità di Endocrinologia, Ospedale di Tinchi-Pisticci, Matera, Italy. roccobruno1@virgilio.it

Salvatore Tumino, Dipartimento di Medicina Clinica e Sperimentale, Università di Catania, Catania, Italy.stumino@unict.it

Graziano Ceresini, Department of Medicine and Surgery, University of Parma, Parma, Italy. ceresini@unipr.it

Marco Centanni, Dipartimento di Scienze e Biotecnologie Medico-Chirurgiche, University of Rome Sapienza, Latina, Italy. marco.centanni@uniroma1.it

Salvatore Monti, Department of Endocrinology, Ospedale S. Andrea, Sapienza Università di Roma, Rome, Italy.salvatore.monti@uniroma1.it

Domenico Salvatore, Department of Clinical Medicine and Surgery, University of Naples "Federico II", Naples, Italy. domenico.salvatore@unina.it

Caterina Mian, Endocrinology Unit, Department of Medicine-DIMED, University-Hospital of Padua, Padua, Italy. caterina.mian@unipd.it

Giovanna Spiazzi, Section of Endocrinology, Diabetes and Metabolism, Department of Medicine, University of Verona, Verona, Italy. giovanna.spiazzi@ospedaleuniverona.it Luca Persani, Department of Clinical Sciences and Community Health, University of Milan; Division of Endocrine and Metabolic Diseases, Istituto Auxologico Italiano IRCCS, 20149 Milan, Italy. luca.persani@unimi.it

Daniele Barbaro, U.O. Endocrinologia, Livorno, ASL Nord Ovest Toscana, Livorno, Italy. danielebarbaro1970@libero.it

Antonio Nicolucci, Center for Outcomes Research and Clinical Epidemiology, Pescara, Italy. nicolucci@coresearch.it 
Sebastiano Filetti, Prof. Department of Internal Medicine and Medical Specialties, University of Rome Sapienza, Rome, Italy.sebastiano.filetti@uniroma1.it

On behalf of the ITCO foundation

Corresponding author and person to whom reprint request should be addressed:

Sebastiano Filetti, Prof

Dipartimento di Medicina Interna e Specialità Mediche

Università di Roma "Sapienza"

Viale del Policlinico 155, 00161, Roma, Italy

Phone: +39-06-499775133

e-mail: sebastiano.filetti@uniroma1.it

Running title DTC guidelines and clinical practice (ITCO\#1).

Key words differentiated thyroid cancer, evidence based guidelines, clinical practice, thyroid surgery, radioiodine remnant ablation

Word count: 3453

Number of tables: 3

Supplementary Tables: 1 
Abstract

Objectives: The goal of evidence-based practice guidelines is to optimize the management of emerging diseases, such as differentiated thyroid cancer (DTC). The aim of this study was to assess therapeutic approaches for DTC in Italy and to see how closely these practices conformed to those recommended in the 2009 American Thyroid Association (ATA) guidelines.

Methods: The Italian Thyroid Cancer Observatory (ITCO) was established to prospectively collect data on thyroid cancers consecutively diagnosed in participating centers (uniformly distributed across the nation). We analyzed data on the initial treatment of all pathologically confirmed DTC cases present in the database from January 1, 2013 (database creation) to January 31, 2016.

Results: 1748 patients (77.2\% females; median age 48.1 years [10-85]) were enrolled in the study. Most ( $n=1640,93.8 \%$ ) were papillary carcinomas (including 84 poorly differentiated/aggressive variants); 6.2\% ( $n=108)$ were follicular and Hürthle cell carcinomas. Median tumor diameter was $11 \mathrm{~mm}$ (1-93 mm). Tumors were multifocal in $613(35 \%)$ and presented extrathyroidal extension in 492 (28\%) cases. Initial treatments included total thyroidectomy (involving one or two procedures) ( $n=1726,98.8 \%$ ) and lobectomy $(n=22,1.2 \%)$. A quarter of the patients that underwent total thyroidectomy had unifocal, intrathyroidal tumors, $\leq 1 \mathrm{~cm}(\mathrm{n}=408 ; 23.6 \%)$. Neck dissection was performed in $40.4 \%$ of the patients ( $29.5 \%$ had central compartment dissection). Radioiodine remnant ablation (RRA) was performed in 1057 (61.2\%) of the 1726 patients who underwent total thyroidectomy: 460 (41.2\%) of the 983 classified by 2009 ATA guideline criteria as low-risk, $570(87.1 \%)$ of the 655 as intermediate-risk, and 82 (93.1\%) of the 88 as high-risk patients $(p<0.001)$. RRA was performed in $44 \%$ of the cases involving multifocal DTCs measuring $\leq 1$ $\mathrm{cm}$.

Conclusions: The treatment approaches for DTCs used in Italy display areas of inconsistency with those recommended by the 2009 ATA guidelines. Italian practices were characterized by underuse of thyroid lobectomy in intrathyroidal, unifocal, $\leq 1 \mathrm{~cm}$ DTCs. The use of RRA was generally consistent with risk-stratified recommendations. However, its frequent use in small DTCs $(\leq 1 \mathrm{~cm})$ that are multifocal persists despite the lack of 
evidence of benefit. These data provide a baseline for future assessments of the impact of international guidelines on DTC management in Italy. These findings also illustrate that the dissemination and implementation of guideline recommendations, and the change in practice patterns, require ongoing education and time. 


\section{Page 7 of 29}

\section{Introduction}

The incidence of differentiated thyroid cancer (DTC) is on the rise, and it now represents the fourth most common cancer in women in Italy (1). Differentiated thyroid cancers generally have an excellent prognosis, with a 5-year survival rate of $98.1 \%(2)$ and low rates of recurrence (3). The challenge today is to develop increasingly individualized treatment strategies with due emphasis on quality of life. A first step toward this goal involves classification of cases according to the risk of recurrence, as proposed by international evidence-based guidelines, such as those issued regularly by the American Thyroid Association (ATA) $(4,5)$. A recent study showed that cases of thyroid cancer managed in accordance with evidence-based guidelines are associated with better outcomes (6). Little information is available on current practices in the treatment of thyroid cancer in Italy and the degree to which they are affected by international evidence-based guidelines.

To address these issues, we recently conducted a retrospective analysis of prospectively collected cases of DTC in the web-based thyroid cancer database set up by the Italian Thyroid Cancer Observatory (ITCO) foundation, a network of physicians, scientists, and patients with interests in thyroid cancer (http://www.itcofoundation.org). The principal aims of the foundation are: 1) to facilitate and promote the development of collaborative research projects on various aspects of thyroid cancer, ranging from its molecular biology and epidemiology to its treatment and long-term management; 2 ) to create a network of thyroid tumor experts willing to share and exchange knowledge and tools for patient care; and 3) to provide scientific support for healthcare policy makers. The ITCO network is expanding steadily. It currently includes 36 centers involved in the care of thyroid cancer patients, which are distributed throughout the Italian national territory. They include tertiary referral centers operating at the national level as well as smaller hospital-based units with local or regional catchment areas.

We retrospectively analyzed ITCO data on 1748 patients with DTC who were operated on in Italy between January 1, 2013 and January 31, 2016. Our aims were to characterize approaches to the treatment of DTC patients used during this period, in terms of the extent of thyroid surgery and the use of radioiodine remnant ablation (RRA), and to 
see how consistent these practices were with the ATA Guidelines for the Management of Thyroid Cancer available at that time (i.e., those issued in 2009) (4).

\section{Methods}

The ITCO's web-based database was opened in 2013 at the Thyroid Cancer Center of the University of Rome La Sapienza (the network's Coordinating Center), and it was subsequently expanded to include data from centers belonging to the newly founded ITCO. It now includes prospectively collected data on over 3500 Italian patients with histologically confirmed diagnoses of differentiated, medullary, or anaplastic thyroid cancer. Cases are included in the database at the time of primary treatment in the reporting ITCO center or, if the patient was operated on elsewhere, at the time he/she began follow-up in the reporting center (Cases are ineligible for inclusion if contact with the reporting center began more than 12 months after primary treatment). Each case record includes information on patient demographics and biometrics, circumstances of the diagnosis, tumor pathology, surgical and radioactive iodine treatments, and the results of periodic follow-up examinations. Sensitive data are encrypted, and the database is managed in an anonymous fashion by a dedicated team of expert biostatisticians, who are also responsible for all statistical analyses of the data.

For the purposes of the present study, we reviewed the database and selected consecutive cases satisfying both of the following inclusion criteria: 1) histological diagnosis of DTC, including papillary, follicular, and poorly differentiated tumors, as well as their variants; 2) registration in the ITCO database between January 1, 2013 and January 31, 2016 (date of publication of the 2015 version of the American Thyroid Association Guidelines for the Management of Thyroid Cancer) (5). Cases were excluded if the database record lacked complete information on the initial treatment or tumor pathology.

For each case, we recorded the following information: Initial treatment: thyroid surgery procedure (total thyroidectomy, thyroid lobectomy, or lobectomy followed by completion thyroidectomy); cervical lymph node dissection (none, central compartment dissection, lateral compartment dissection, central and lateral compartment dissection). For patients who had undergone total thyroidectomy, we also recorded the use of RRA (performed or omitted). Risk of recurrence: The risk category was calculated by the study 


\section{Page 9 of 29}

team in accordance with the 2009 ATA Guidelines (4). Classification was based on the data available at the time of the initial treatment, with the following exceptions: 1 ) In cases involving lobectomy followed by completion thyroidectomy, we considered pathologic data on tissues collected during both surgical procedures; 2 ) in patients who underwent RRA, post-treatment whole body scintigraphy findings were excluded from risk assessments. Aggressive PTCs: These included solid, insular, tall-cell, columnar-cell, hobnail-cell, sclerosing, and poorly differentiated variants as well as PTCs with foci of invasion of intratumoral vessels. Follicular thyroid cancers (FTC) and Hürthle cell carcinomas: These tumor types were combined and analyzed separately from other DTCs. FTCs were classified as widely invasive (presence of extensive vascular or capsular invasion or minimally invasive (absence of such extensive invasion) (7).

\section{Statistical analysis}

In descriptive analysis, continuous variables were expressed as medians with interquartile ranges (IQR) and nominal variables as numbers and percentages. Differences between categoric variables were calculated with the chi-square test; differences between continuous variables were assessed with the Mann Whitney test. All statistical analyses were performed with SAS software (SAS Institute, Inc.).

\section{Results}

We analyzed a total of 1748 cases of DTC enrolled in the ITCO database between January 1, 2013 and January 31, 2016. The cases were reported by a total of 28 ITCO centers (Supplementary Table 1). The population characteristics are summarized in Table 1. The median age at diagnosis was 48.1 years (range 10-85), and the majority of patients were women. Over $90 \%$ of the tumors were PTCs - classic forms [n=1556] or aggressive variants [ $n=84]$. The remaining $108(6.2 \%)$ were FTCs $(n=80)$ or Hürthle cell carcinomas $(n=27)$.

\section{Surgical management}

On the basis of strong evidence, the 2009 ATA guidelines recommended total or near-total thyroidectomy for all thyroid cancers except for unifocal intrathyroidal tumors with maximum diameters of $\leq 1 \mathrm{~cm}$. In the ITCO cohort, total or near-total thyroidectomy 
was performed on almost all patients (97.9\%), including 408 with unifocal intrathyroidal tumors measuring $\leq 1 \mathrm{~cm}$. Thirty-six (2.1\%) patients were treated with thyroid lobectomy, but 14 of these patients later had completion thyroidectomies.

Lymph node dissection was performed in 706 patients (40.4\% of the total cohort) and involved the central compartment alone in 516 patients, both central and lateral compartments in 160 , and the lateral compartment alone in 30 . Lymph node metastases were found in 383 (54\%) of the 706 patients who underwent lymph node neck dissection and in 157 (83\%) of the 190 that underwent lateral neck dissection.

The 22 patients treated with thyroid lobectomy alone were older (median age 50.7 years). One had a Hürthle cell carcinoma measuring $28 \mathrm{~mm}$; the other 21 had PTCs. Most PTCs in this group were classified as low-risk (19/21, 86.4\%) according to the 2009 ATA guidelines. In the remaining two cases (13.6\%), the recurrence risk was considered intermediate owing to the metastatic involvement of a single central-compartment lymph node in one and evidence of vascular invasion in the other. All but four of the 22 patients in this group had unifocal tumors, and in all but six, maximum tumor diameters measured $\leq 1 \mathrm{~cm}$ (range $1-15 \mathrm{~mm}$ ).

\section{Use of RRA}

Of the 1726 total thyroid resections (including those preceded by lobectomy), well over half $(1057,61.2 \%)$ were followed by RRA. Patients in this subgroup were significantly younger than those who did not undergo ablation (median: 46.3 years [IQR 36.5-57.4] vs. 51.1 [41.0-60.3]; $p<0.001)$. RRA was done after $41.2 \%$ (405/983) of the thyroidectomies performed on patients considered at low risk for recurrence, $87.1 \%$ (570/655) of those performed on patients at intermediate risk, and 93.1\% (82/88) of those done in the highrisk group $(p<0.001)$. The median dose of radioiodine administered to low-risk patients was $50 \mathrm{mCi}$ (IQR 50-100), and it was significantly lower than the median doses recorded for the intermediate- and high-risk groups (100 mCi, IQR 70 -100 and $100 \mathrm{mCi}$, IQR 100 100 respectively) ( $p<0.001$ ). This observation is consistent with the 2009 ATA recommendation (no. 36), which advocates (on the basis of fair evidence) the use of the lowest activity of radioiodine capable of producing successful remnant ablation, especially in low-risk patients. Preparation for RRA involved thyroid hormone withdrawal in 636 
(60.2\%) of the 1045 cases in which this information was available. Recombinant human TSH (rhTSH) was used in the remaining 409 (38.7\%) cases-that is, 207 (51.1\%) of the 405 low-risk patients who underwent RRA, 176 (30.9\%) of the 570 in the intermediate-risk group, and 26 (31.7\%) of the 82 at high-risk for recurrence. The guidelines specified that, on the basis of strong evidence, either method could be used to prepare patients for RRA.

\section{Consistency with the 2009 ATA Guidelines}

These data were then analyzed in light of the ATA's 2009 recommendations on the use of RRA in subgroups of patients within each risk category (4). The 1619 patients undergoing thyroidectomies for PTCs (Table 2) and the 107 patients who had thyroidectomies for FTCs or Hürthle cell carcinomas (Table 3) were analyzed separately. The results are presented along with the recommendations contained in both the 2009 and 2015 ATA guidelines. The former were used for the primary analysis since they were the reference point during the treatment period considered in the study (2013-2016); practices in the cohort were also analyzed to see how they corresponded with the 2015 recommendations, which were published after the study period.

Patients with PTCs. As shown in Table 2, in 2009 the ATA issued an unequivocal recommendation against the use of RRA only for low-risk patients harboring intrathyroidal PTCs with maximum diameters of $\leq 1 \mathrm{~cm}$ and nonaggressive histology, even when multiple lesions are present. This decision was based on fair evidence showing that this intervention does not improve important health outcomes or that its potential harms outweigh its expected benefits. In the ITCO cohort, RRA was performed on fewer than one out of five patients with unifocal PTCs $\leq 1 \mathrm{~cm}(16.8 \%)$ but almost half of those whose microcarcinomas were multifocal (44\%).

At the other end of the spectrum, unequivocal recommendations for the use of RRA (albeit based on evidence with different strength ratings) were made for all high-risk PTC patients, as well as for specific subgroups at intermediate- or low-risk of recurrence. In the ITCO cohort, $96.3 \%$ (53/55) of the high-risk patients were treated in accordance with the strong recommendation for RRA. In the intermediate-risk group, the ATA recommendation for RRA in patients with lymph node metastases was based solely on expert opinion, but it nonetheless corresponded to the treatment used in the vast majority 
(91.6\%; $241 / 263)$ of cases. Use of RRA appeared to be unrelated to the location and number of the metastatic lymph nodes: it was done in $87.7 \%$ of patients with metastatic involvement of $<5$ central-compartment lymph nodes, $83.3 \%$ of those with involvement of $\geq 5$ central compartment nodes, and $87.6 \%$ of those metastatic disease restricted to the lateral compartments. In the low-risk category, RRA was recommended only for patients with tumors larger than $4 \mathrm{~cm}$. Although this decision was based on fair evidence of benefit, treatment in the ITCO cohort coincided with this recommendation in only $70.6 \%(12 / 17)$ of cases.

For the PTC patients in the remaining low- and intermediate-risk subgroups, the ATA recommended selective use of RRA, based on the clinical judgment of the practitioner and consideration of "risk-modifying" factors, such as the presence of multiple tumor foci, aggressive histology, or vascular invasion. In the low-risk category, selective use of RRA was advised for tumors ranging in size from $>1 \mathrm{~cm}$ to $4 \mathrm{~cm}$. In the ITCO cohort, RRA was done in most cases of this type, although the frequency was slightly higher in the presence of multifocal disease (65.6\% vs. $57.7 \%$ of patients with unifocal disease). As for the use of RRA in patients at intermediate risk, the frequency ranged from $74.9 \%$ in patients with minimal extrathyroidal extension (microscopically evident) to over $90 \%$ in the presence of aggressive histology.

Patients with FTCs or Hürthle cell carcinomas. As shown in Table 3, the ATA unequivocally recommended RRA for FTCs that were widely invasive and for Hürthle cell carcinomas. Both decisions were based on expert opinion alone. Use of RRA in the ITCO cohort was consistent with these recommendations in all cases of widely invasive FTC and in most cases of Hürthle cell carcinomas (77.8\%; 21/27). Selective use was recommended for minimally invasive FTCs. RRA was used to treat over $80 \%$ of patients with such tumors (as well as two others with FTCs whose invasiveness was not classified).

Multivariate analysis showed that the likelihood of undergoing RRA increased significantly with recurrence risk class (OR 10.2 and 17.8 for intermediate- and high-risk patients, 95\% $\mathrm{Cl} 7.80-13.21$ and 7.70-41.20 respectively) and decreased with advancing age (OR 0.93, 95\% Cl 0.90-0.96, $p<0.0001)$. 


\section{Discussion}

The aim of this study was to assess surgical approaches and use of RRA in Italy for treatment of DTC and to see how closely these practices conformed to those advocated by the ATA in 2009. The treatment period analyzed-January 2013 through January 2016was considered appropriate for assessing the effects of the 2009 ATA guidelines in Italy. By October 2009, these guidelines had been translated into Italian and were available for consultation by the Italian medical community. On the whole, the ATA recommendations reflected a clear preference for more conservative management of selected DTCs in terms of surgery and RRA. In both cases, the real-life clinical practice we documented in Italy during this period was somewhat less selective than recommended.

This was especially true of surgical management. The DTCs were almost invariably treated with total or near-total thyroidectomy, regardless of the estimated risk for recurrence. Overuse of total thyroidectomy in the ITCO cohort may be due in part to the high prevalence of bilateral multinodular thyroid disease in Italy (10). Unfortunately, the ITCO database records contain no information about nodules in the lobe without cancer that could confirm or disprove this hypothesis. In experienced hands, the rate of permanent complications of total thyroidectomy can be very low (11). A frequent preference for thyroidectomy has also been documented in the United States. Recent surveys indicate that up to $60 \%$ of physicians caring for thyroid cancer patients and involved in the National Cancer Database studies still advocate total/near-total thyroidectomy for small tumors, in spite of recommendations favoring lobectomy in these cases $(12,13)$. This preference was also strongly associated with a tendency to overuse RRA in low-risk patients (12).

There was a more evident trend towards selective use of RRA. Ablation was restricted to roughly $60 \%$ of the patients who underwent thyroidectomy, including all those at high-risk for recurrence. Its use in the low- and intermediate-risk cases was generally associated with the presence of lymph node metastases and/or aggressive histotypes, as recommended. However, its frequent use for microPTCs that were multifocal (44\% vs. $16.8 \%$ of unifocal microPTCs) was in clear contrast with the 2009 ATA guidelines. This trend may stem in part from some reports indicating that these tumors are more likely to recur than their unifocal counterparts $(14,15)$, although other studies have 
failed to confirm this difference, even among microPTCs harboring BRAF mutations (16). Differential risk notwithstanding, RRA has been shown to have virtually no impact on the likelihood of microPTC recurrence $(14,15)$. Again, the preferences observed in the ITCO cohort have also been documented in the United States. Few of the American thyroid surgeons surveyed felt that RRA was appropriate for unifocal PTCs measuring $<1 \mathrm{~cm}$, but up to $40 \%$ advocated its use when the microPTC was multifocal (12).

Many low-risk patients in the ITCO cohort were still being treated with high activities of ${ }^{131} \mathrm{I}$. However, the median ${ }^{131} \mathrm{I}$ dose used in the low-risk subgroup was lower (50 $\mathrm{mCi})$ than those administered in intermediate- and high-risk cases $(100 \mathrm{mCi})$, which is consistent with 2009 ATA recommendations and with increasing awareness of the higher rate of side effects associated with high ${ }^{131}$ I activities (17). The efficacy and safety of loweractivity of radioiodine $(30 \mathrm{mCi})$ in low risk patients were also confirmed during the study period by the results of two European randomized clinical trials (ESTIMABL and HiLo) $(8,9)$. Recombinant human TSH, rather than thyroid hormone withdrawal, was used for RRA preparation in nearly a half the low-risk patients. The use of rhTSH is consistent with the growing concern with quality of life and the need to avoid whenever possible unpleasant symptoms provoked by withdrawal of thyroid hormone therapy (18), and the efficacy of this approach has been re-confirmed by the results of the ESTIMABL and HiLo trials $(8,9)$. The heterogeneity of its use in real-life clinical practice may also reflect lack of uniformity in terms of local, regional, or national healthcare budgets and/or the availability of tools and/or technology required for guideline implementation. Such factors might partly explain the widespread use of hormone withdrawal in our cohort. The cost of rhTSH is undeniably a consideration. However, cost-effectiveness data published a year before the treatment period considered in our study had already demonstrated that the higher cost of rhTSH could be outweighed by savings related to the shorter hospital stays it allows (19). This is an important consideration in a country like Italy, where RRA is always performed on an in-patient basis due to strict policies on radioprotection and the cost of in-patient procedures are covered by the public healthcare system. Healthcare budgets and the number of nuclear medicine units can vary considerably between one region of Italy and another. 
Implementation of practice guidelines also requires time. Publication is inevitably followed by a "latency period" during which the recommendations have to be read, considered, and discussed by clinicians, healthcare policymakers, and patient advocacy groups, and this lag can be particularly significant for recommendations that fall into "gray zones" where the evidence is less clear-cut.

In addition, for international guidelines like those of the ATA, an accurate and validated translation of the recommendations must also be produced to ensure their widespread diffusion throughout the local community, where knowledge and understanding of English vary. Language barriers are likely to be lower in certain settings, such as metropolitan and/or academic healthcare facilities, and this might favor earlier access to and understanding of recommendations drafted in English. Unfortunately, we were not able to analyze ATA guideline implementation in our cohort by treatment facility type or location because the reporting center and the center where the patient was treated were not always the same.

The results of our study were also analyzed in light of evolving trends as reflected in the 2015 ATA guidelines on DTC management. In most respects, the 2015 edition confirms or strengthens the positions adopted in the 2009 version. For example, the recommendation for the use of thyroid lobectomy-apparently rejected by most Italian thyroid surgeons, even for treatment of microscopic unifocal intrathyroidal tumors-was broadened in the 2015 edition to include all unifocal intrathyroidal tumors up to $4 \mathrm{~cm}$ in size. The ATA continues to strongly recommend RRA in patients at high risk for recurrence, and this position is rarely disputed. In contrast, its persistent rejection of RRA for intrathyroidal microcarcinomas without aggressive histology, including those that are multifocal, is, as we have seen, another area in which real-life and recommended practices display substantial divergence in Italy [Table 2] and elsewhere (12)-(13). It will be interesting to see whether and how practices in the ITCO network change in light of the increasingly strong evidence supporting this "no" in the 2015 guidelines. In cases characterized by intermediate-risk, RRA use rates in Italy were high (Table 2). This trend was fully in keeping with the 2009 guidelines on patients with lymph-node metastases in general, but less so with the 2015 recommendations on this group. The latter are more 
articulated, and RRA is no longer considered mandatory in certain subgroups (e.g., patients with fewer than five microscopic nodal metastases).

In summary, our study reveals discrete areas in the management of DTC in which daily clinical practice seems to diverge from the internationally endorsed guidelines issued by the ATA. Extensive surgical treatment is still widely used for DTC in Italy, regardless of disease stage and risk status. Use of radioiodine remnant ablation appeared to be more consistent with the 2009 ATA risk stratification. These data provide a useful baseline for future analyses of ITCO data aimed at assessing the impact of international guidelines on real-life clinical management of DTCs in Italy. These findings also illustrate that the dissemination and implementation of guideline recommendations, and the change in practice patterns, require ongoing education and time.

Acknowledgments. LL and GG contributed to this paper as recipient of the PhD program of Biotechnologies and Clinical Medicine of the University of Rome, "Sapienza".

We thank all the members of the ITCO foundation:

Ancona: Alessia Smerilli, Augusto Taccaliti, Bari: Vincenzo Triggiani, Giuseppina Renzulli, Brescia: Maria Beatrice Panarotto, Cagliari: Stefano Mariotti, Fabiana Pani, Catania:

Simona Quartararo, Dario Tumino, Ferrara: Ettore Degli Uberti, La Sepzia: Laura Camerieri, Mario Cappagli, Latina: Maria Giulia Santaguida, Livorno: Paola Lapi, Matera: Antonella Carbone,

Milano: Biagio Cangiano, Chiara Cacciatore, Carla Colombo, Simone De Leo, Andrea Lania, Napoli: Maria Grazia Chiofalo, Luciano Pezzullo, Tommaso Porcelli, Novara: Chiara Mele, Loredana Pagano, Padova: Simona Censi, Palermo: Adele Maniglia, Andrea Attard, Parma: Michela Marina, Pisa: Nadia Caraccio, Poupak Fallahi, Filippo Niccolai, Giuseppe Pasqualetti, Armando Patrizio, Roma: Anna Crescenzi, Laura Ciotti, Rossella Del Toro, Francesca lanni, Angelo Lauria, Valeria Loiacono, Pietro Locantore, Cristiano Lomonaco, Sara Nazzarena Morgante, Valeria Nestola, Serena Elisa Tempera, Giuseppe Ronga, Vincenzo Toscano, Emanuela Traini, San Giovanni Rotondo: Leonardo D’Aloiso, Michela Massa, Siena: Maria Grazia Castagna, Raffaella Forleo, Fabio Maino, Ezio Ghigo, Stefania Corvisieri, Verona: Chiara Conforti, Amalia Robustelli. 
ठิ. $\quad$ Page 17 of 29

들

롤

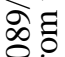

을

o

길

\#节咅

0.

$\exists \geq$

त्वृ

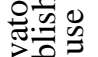

흘펴

음후

¿

हैं

站.

일

트응

즌

퐁융

ت뭉

증

․․요

రृ.

-

능음

웜유.

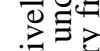

등을

넝국

율

प्त्व

으를

的涼

일

$\begin{array}{ll}17 & 17\end{array}$

Writing support was provided by Marian Everett Kent, BSN, and funded by the Fondazione ब Umberto Di Mario.

สิ

ํ.

站突

Disclosure Statement. Authors have nothing to disclose 


\section{References}

1. Group AW 2014 Italian cancer figures, report 2014: Prevalence and cure of cancer in Italy. Epidemiol Prev 38:1-122.

2. Institute NC. Surveillance, Epidemiology and End Results Program, Cancer Stat Facts. https://seer.cancer.gov/. (Last accessed on 02 May 2017, 2017).

3. Durante C, Montesano T, Torlontano M, Attard M, Monzani F, Tumino S, Costante G, Meringolo D, Bruno R, Trulli F, Massa M, Maniglia A, D'Apollo R, Giacomelli L, Ronga G, Filetti S, Group PS 2013 Papillary thyroid cancer: time course of recurrences during postsurgery surveillance. J Clin Endocrinol Metab 98:636-642.

4. Cooper DS, Doherty GM, Haugen BR, Hauger BR, Kloos RT, Lee SL, Mandel SJ, Mazzaferri EL, Mclver B, Pacini F, Schlumberger M, Sherman SI, Steward DL, Tuttle RM, Cancer ATAAGToTNaDT 2009 Revised American Thyroid Association management guidelines for patients with thyroid nodules and differentiated thyroid cancer. Thyroid 19:1167-1214.

5. Haugen BR, Alexander EK, Bible KC, Doherty GM, Mandel SJ, Nikiforov YE, Pacini F, Randolph GW, Sawka AM, Schlumberger M, Schuff KG, Sherman SI, Sosa JA, Steward DL, Tuttle RM, Wartofsky L 20162015 American Thyroid Association Management Guidelines for Adult Patients with Thyroid Nodules and Differentiated Thyroid Cancer: The American Thyroid Association Guidelines Task Force on Thyroid Nodules and Differentiated Thyroid Cancer. Thyroid 26:1-133.

6. Goffredo P, Roman SA, Sosa JA 2014 Have 2006 ATA practice guidelines affected the treatment of differentiated thyroid cancer in the United States? Thyroid 24:463471.

7. DeLellis, R, Lloyd R, Heitz P, C. E 2004 WHO Classification of Tumours, Pathology and Gene . tics of Tumours of Endocrine Organs. 3rd ed. ed.

8. Schlumberger M, Catargi B, Borget I, Deandreis D, Zerdoud S, Bridji B, Bardet S, Leenhardt L, Bastie D, Schvartz C, Vera P, Morel O, Benisvy D, Bournaud C, Bonichon F, Dejax C, Toubert ME, Leboulleux S, Ricard M, Benhamou E, Trial TdlTRNftESAE 2012 Strategies of radioiodine ablation in patients with low-risk thyroid cancer. $\mathrm{N}$ Engl J Med 366:1663-1673.

9. Mallick U, Harmer C, Yap B, Wadsley J, Clarke S, Moss L, Nicol A, Clark PM, Farnell K, 
McCready R, Smellie J, Franklyn JA, John R, Nutting CM, Newbold K, Lemon C, Gerrard G, Abdel-Hamid A, Hardman J, Macias E, Roques T, Whitaker S, Vijayan R, Alvarez P, Beare S, Forsyth S, Kadalayil L, Hackshaw A 2012 Ablation with low-dose radioiodine and thyrotropin alfa in thyroid cancer. N Engl J Med 366:1674-1685.

10. Durante C, Costante G, Lucisano G, Bruno R, Meringolo D, Paciaroni A, Puxeddu E, Torlontano M, Tumino S, Attard M, Lamartina L, Nicolucci A, Filetti S 2015 The natural history of benign thyroid nodules. JAMA 313:926-935.

11. Rosato L, Avenia N, Bernante P, De Palma M, Gulino G, Nasi PG, Pelizzo MR, Pezzullo L 2004 Complications of thyroid surgery: analysis of a multicentric study on 14,934 patients operated on in Italy over 5 years. World J Surg 28:271-276.

12. Haymart MR, Banerjee M, Yang D, Stewart AK, Doherty GM, Koenig RJ, Griggs JJ 2013 The relationship between extent of thyroid cancer surgery and use of radioactive iodine. Ann Surg 258:354-358.

13. Haymart MR, Banerjee M, Yang D, Stewart AK, Sisson JC, Koenig RJ, Doherty GM, Griggs JJ 2013 Variation in the management of thyroid cancer. J Clin Endocrinol Metab 98:2001-2008.

14. Ross DS, Litofsky D, Ain KB, Bigos T, Brierley JD, Cooper DS, Haugen BR, Jonklaas J, Ladenson PW, Magner J, Robbins J, Skarulis MC, Steward DL, Maxon HR, Sherman SI 2009 Recurrence after treatment of micropapillary thyroid cancer. Thyroid 19:10431048.

15. Hay ID, Hutchinson ME, Gonzalez-Losada T, Mclver B, Reinalda ME, Grant CS, Thompson GB, Sebo TJ, Goellner JR 2008 Papillary thyroid microcarcinoma: a study of 900 cases observed in a 60-year period. Surgery 144:980-987; discussion 987988.

16. Tallini G, de Biase D, Durante C, Acquaviva G, Bisceglia M, Bruno R, Bacchi Reggiani ML, Casadei GP, Costante G, Cremonini N, Lamartina L, Meringolo D, Nardi F, Pession A, Rhoden KJ, Ronga G, Torlontano M, Verrienti A, Visani M, Filetti S 2015 BRAF V600E and risk stratification of thyroid microcarcinoma: a multicenter pathological and clinical study. Mod Pathol 28:1343-1359.

17. Lamartina L, Durante C, Filetti S, Cooper DS 2015 Low-risk differentiated thyroid cancer and radioiodine remnant ablation: a systematic review of the literature. J 
Clin Endocrinol Metab 100:1748-1761.

18. Schroeder PR, Haugen BR, Pacini F, Reiners C, Schlumberger M, Sherman SI, Cooper DS, Schuff KG, Braverman LE, Skarulis MC, Davies TF, Mazzaferri EL, Daniels GH, Ross DS, Luster M, Samuels MH, Weintraub BD, Ridgway EC, Ladenson PW 2006 A comparison of short-term changes in health-related quality of life in thyroid carcinoma patients undergoing diagnostic evaluation with recombinant human thyrotropin compared with thyroid hormone withdrawal. J Clin Endocrinol Metab 91:878-884.

19. Borget I, Remy H, Chevalier J, Ricard M, Allyn M, Schlumberger M, De Pouvourville G 2008 Length and cost of hospital stay of radioiodine ablation in thyroid cancer patients: comparison between preparation with thyroid hormone withdrawal and thyrogen. Eur J Nucl Med Mol Imaging 35:1457-1463. 


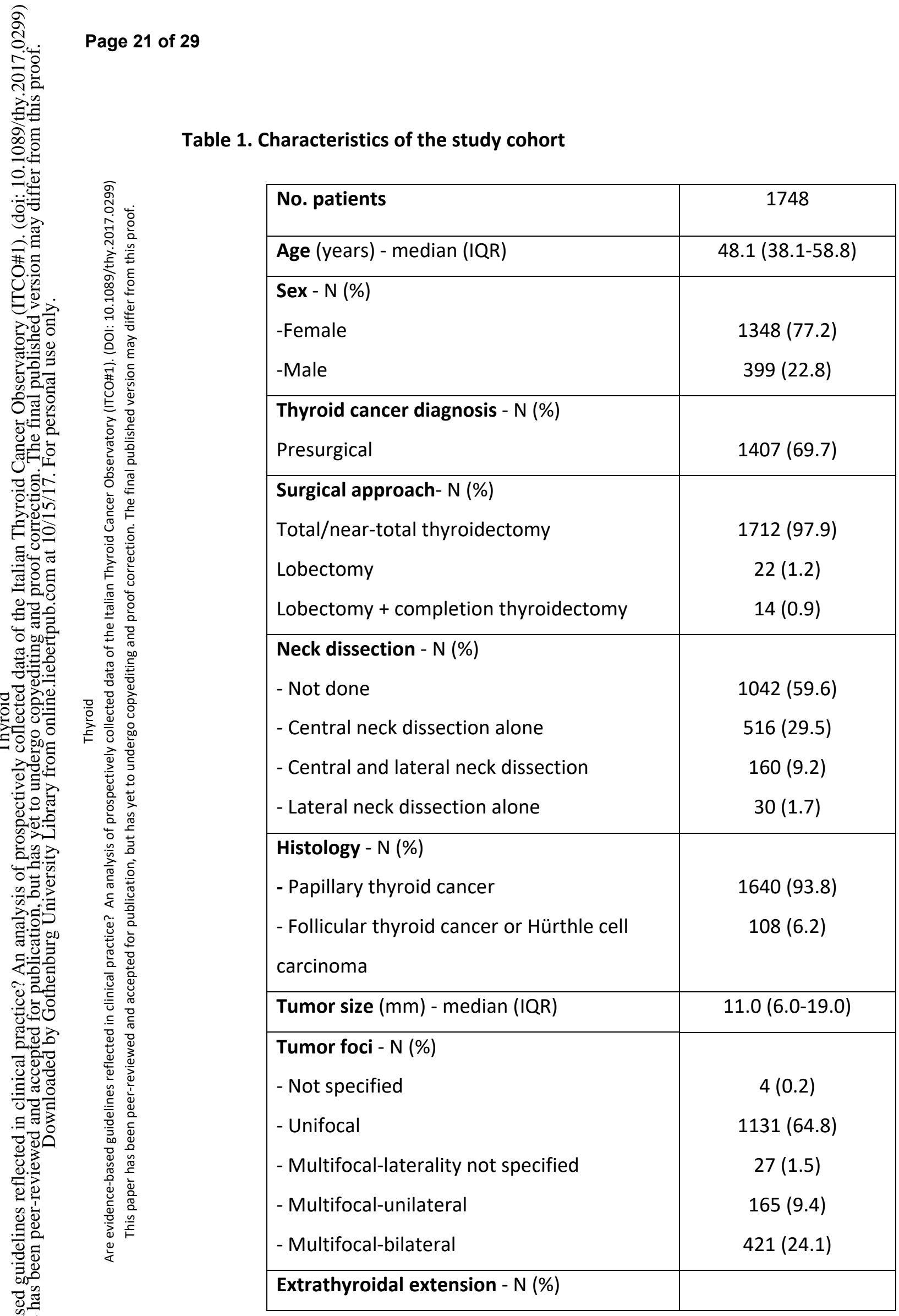


- None

- Microscopic (T3)

1256 (71.9)

435 (24.9)

- Macroscopic (T4a)

53 (3.0)

- Macroscopic (T4b)

$4(0.2)$

Lymph node status - N (\%)

$\mathrm{Nx}$

NO

N1a

N1b

$233(13.3)$

Number of metastatic lymph nodes median (IQR)

2009 ATA risk - N (\%)

- Low

1002 (57.3)

- Intermediate

$657(37.6)$

- High

$89(5.1)$

Abbreviations: ATA: American thyroid association; IQR: inter quartile range 


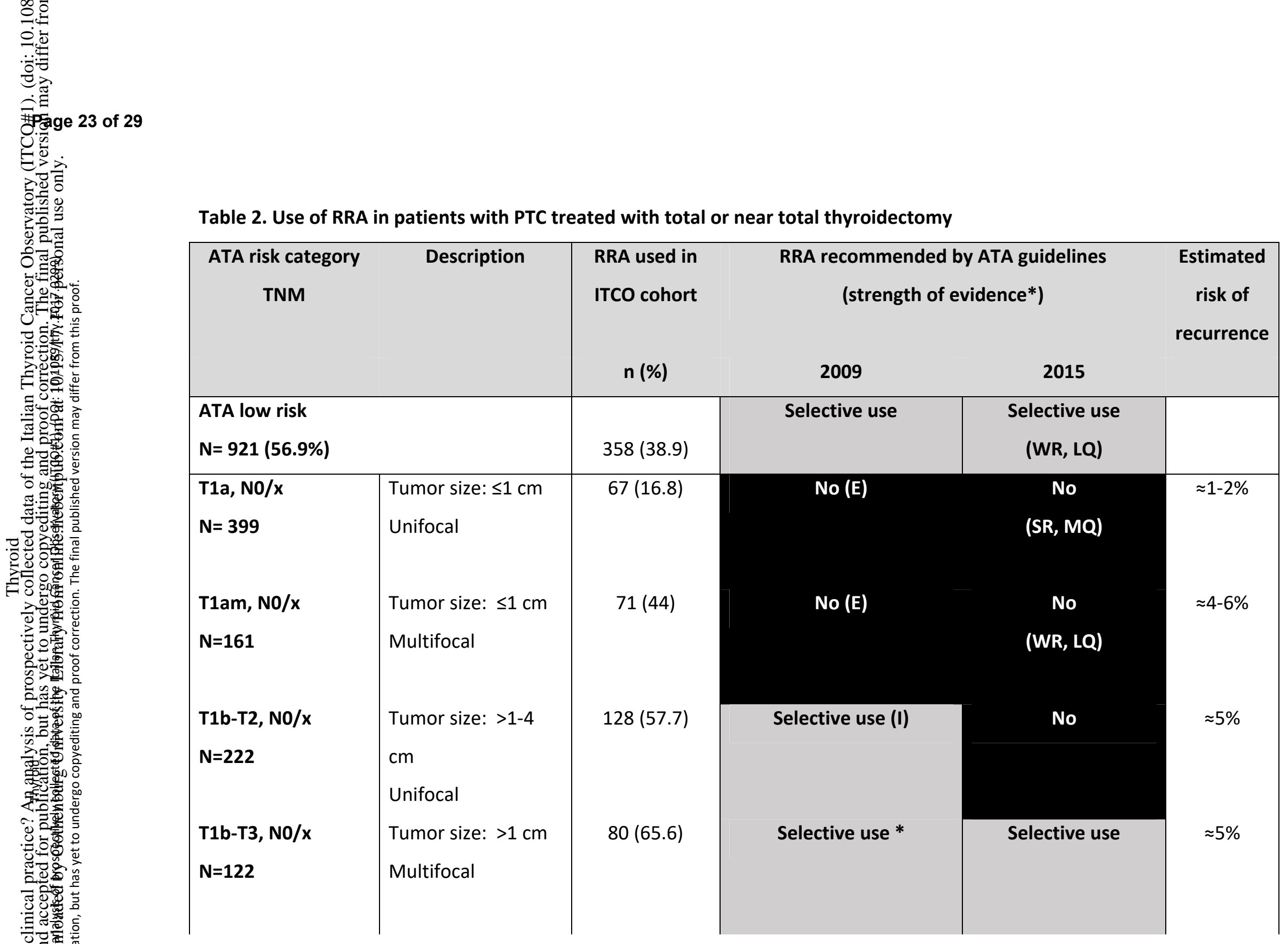




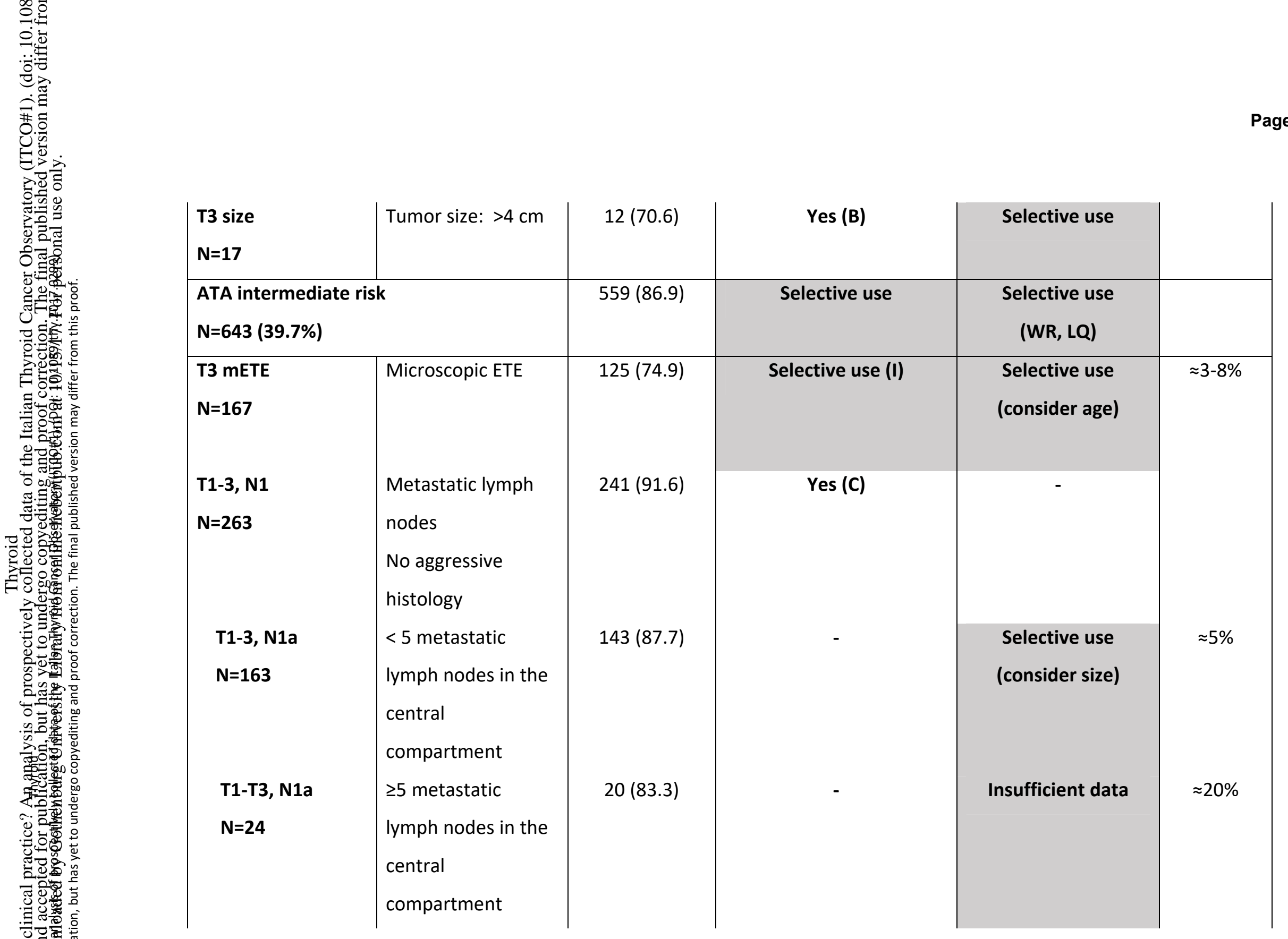




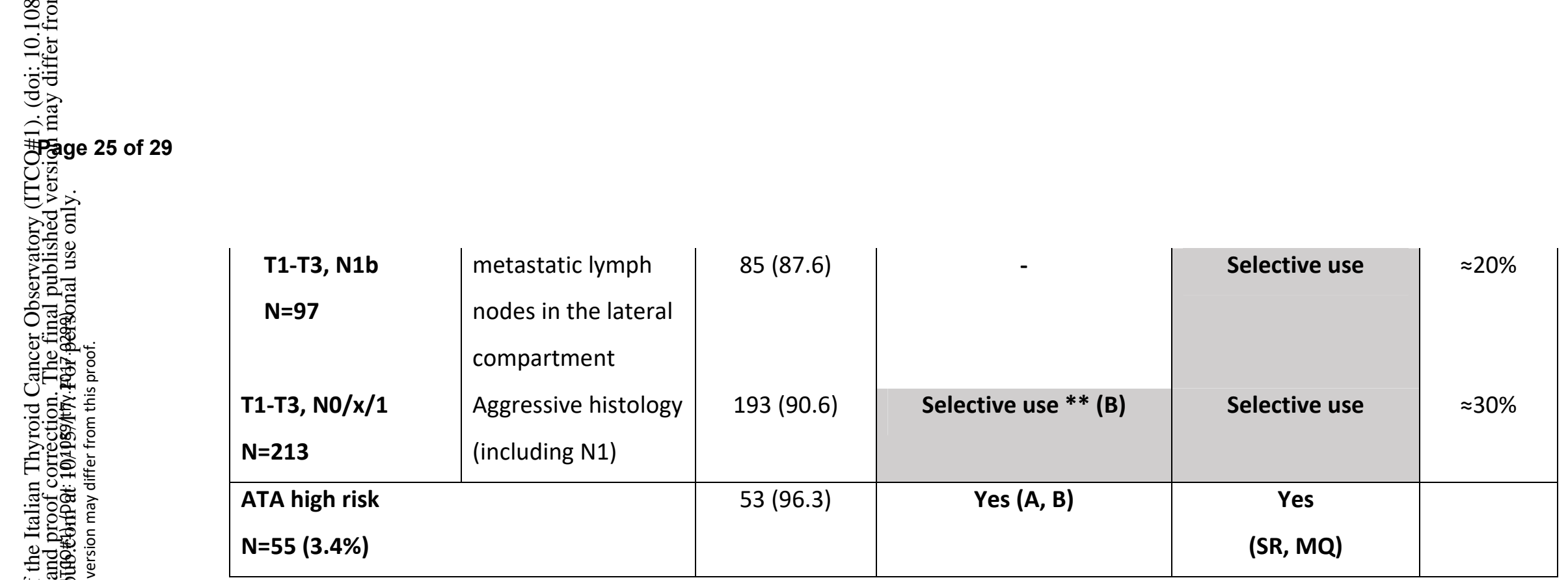

Abbreviations: ATA: American Thyroid Association; RRA: radioiodine remnant ablation; PTC: papillary thyroid cancer; ETE: extrathyroidal extension.

*Strength of evidence: A: strong evidence for; B: fair evidence for; C: expert opinion favors; E: fair evidence against; I: neither for nor against; WR: weak recommendation; LQ low-quality evidence; MQ: moderate-quality evidence; SR: strong recommendation.

**Modifying factors: aggressive histology, multifocality and vascular invasion. 
Table 3. Use of RRA in patients with FTC or Hürthle cell carcinoma treated with total or near-total thyroidectomy

\begin{tabular}{|c|c|c|c|c|}
\hline \multirow[t]{2}{*}{$\begin{array}{c}\text { ATA risk } \\
\text { TNM }\end{array}$} & \multirow{2}{*}{$\begin{array}{l}\text { RRA used in ITCO } \\
\text { cohort } \\
\text { n (\%) }\end{array}$} & \multicolumn{2}{|c|}{$\begin{array}{l}\text { RRA recommended by ATA } \\
\text { guidelines } \\
\text { (strength of evidence) }\end{array}$} & \multirow[t]{2}{*}{$\begin{array}{c}\text { Estimated } \\
\text { risk of } \\
\text { recurrence }\end{array}$} \\
\hline & & 2009 & 2015 & \\
\hline $\begin{array}{c}\text { FTC/HCC } \\
\text { N=107 }\end{array}$ & 87 (81.3) & & & \\
\hline $\begin{array}{l}\text { Minimally } \\
\text { invasive } \\
\mathrm{N}=59\end{array}$ & $48(81.4)$ & Selective use & Selective use & $\approx 2-3 \%$ \\
\hline $\begin{array}{l}\text { Widely } \\
\text { invasive } \\
\mathrm{N}=16\end{array}$ & $16(100)$ & Yes (C) & Yes & $\approx 30-55 \%$ \\
\hline $\begin{array}{l}\text { Hürthle } \\
\text { cell } \\
\text { carcinoma } \\
\mathrm{N}=\mathbf{2 7}\end{array}$ & $21(77.8)$ & Yes (C) & - & \\
\hline $\begin{array}{l}\text { FTC NOS } \\
N=5\end{array}$ & $2(40)$ & - & - & \\
\hline
\end{tabular}


Page 27 of 29

\section{Supplementary Table 1. List of the ITCO centers involved in the study}

\section{Region}

\section{North of Italy}

Bologna:

- Ospedale di Bentivoglio, Department of Endocrinology, Unità

Operativa Semplice

Brescia:

- Università degli Studi di Brescia, Spedali Civili, Nuclear Medicine Unit

Ferrara:

- Azienda Ospedaliero Universitaria S. Anna, Endocrine Unit

Livorno:

- Azienda Sanitaria Locale Nord Ovest Toscana, Unità Operativa di

Endocrinologia

Milano:

- IRCCS Istituto Auxologico Italiano, Division of Endocrine and Metabolic

Diseases

- Università degli Studi di Milano, Department of Pathophysiology and

Transplantation

Novara:

- Università del Piemonte Orientale, Endocrinology, Department of

Translational Medicine

Padova:

- University-Hospital of Padua, Endocrinology Unit, Department of

Medicine-DIMED

Parma:

- University of Parma, Department of Medicine and Surgery

Torino:

- University of Turin, Department of Medical Sciences,

- University of Turin, Department of Oncology, Division of Endocrinology and Metabolism, Humanitas-Gradenigo Hospital 
- Mauriziano Umberto I Hospital, Division of Endocrinology, Diabetology

and Metabolism

- University of Turin, Division of Endocrinology, Diabetology and

Metabolism, Department of Medical Sciences, Molinette Hospital,

A.O.U. Città della Salute e della Scienza di Torino

Verona:

- University of Verona, Section of Endocrinology, Diabetes and

Metabolism, Department of Medicine

\section{Center of Italy}

Latina:

- University of Rome Sapienza, Polo Pontino, Dipartimento di Scienze e

Biotecnologie Medico-Chirurgiche

Perugia:

- University of Perugia, Department of Medicine

Pisa:

- University of Pisa, Geriatrics Unit, Department of Clinical \&

Experimental Medicine

- University of Pisa, Department of Clinical and Experimental Medicine

Roma:

- University of Rome Sapienza, Department of Internal Medicine and

Medical Specialties, Policlinico Umberto I

- University of Rome Sapienza, Department of Nuclear Medicine,

Policlinico Umberto I

- University of Rome Sapienza, Ospedale S. Andrea

- Fondazione Policlinico Gemelli, Catholic University, Division of

Endocrinology and Metabolic Diseases and Division of Endocrine Surgery

\section{South of Italy}

Catania:

- University of Catania, Dipartimento di Medicina Clinica e Sperimentale

Matera: 
ठิ. $\quad$ Page 29 of 29

드윰

- Ospedale di Tinchi-Pisticci, Unità di Endocrinologia

Napoli:

- University of Naples Federico II, Department of Clinical Medicine and

Surgery

Palermo:

- Cervello Hospital, Division of Endocrinology

Salerno:

- Division of Endocrinology

San Giovanni Rotondo - Foggia:

- Ospedale Casa Sollievo della Sofferenza-IRCCS, Department of Medical

Science

Total 